\title{
microRNAs and response to statins in patients with hypercholesterolemia: from basic research to precision medicine
}

\author{
Tomás Zambrano ${ }^{1,2}$ \& Luis A Salazar*,1 \\ ${ }^{1}$ Center of Molecular Biology \& Pharmacogenetics, Scientific \& Technological Bioresource Nucleus (BIOREN), Universidad de La \\ Frontera, Temuco, Chile \\ ${ }^{2}$ Department of Medical Technology, Faculty of Medicine, Universidad de Chile, Santiago, Chile \\ *Author for correspondence: Tel.: + 5645259 6724; luis.salazar@ufrontera.cl
}
' $\boldsymbol{\sigma}_{\text {is }}$ the massive task of making precision medicine a reality completely within our reach? To know what we do not know, a large road remains ahead, as a growing number of continuous breakthroughs demonstrate us that accumulated knowledge so far can only expand, especially in the postgenomic era"

First draft submitted: 10 April 2018; Accepted for publication: 11 April 2018; Published online: 22 May 2018

Keywords: hypercholesterolemia - microRNAs - personalized medicine • pharmacoepigenetics - pharmacogenomics • statins

Basic research matters. It not only matters, but becomes pivotal for subsequent findings based on the earliest reports, as we will observe throughout this editorial. Since their first description in the nematode $C$. elegans more than two decades ago [1], miRNAs have been in a constantly increasing spotlight for many years. These molecules are short, noncoding, single-stranded RNAs that function as a gene silencing mechanism by complementary binding to an incredibly diverse number of mRNA sequences, thereby, suppressing protein expression of their target mRNAs, and depicting a very complex intracellular fine-tuning that, when impaired, may alter profoundly numerous cellular processes involved in several physiological and pathological conditions. In recent times, the interest in miRNA research has only gained momentum as a deeper knowledge into their practical applications has been obtained, especially when uncovering their remarkable stability in several body fluids, establishing a novel and important role as noninvasive specific biomarkers for many pathologies which made them an obvious and highly attractive investigation focus for rapidly advancing research areas that encompass major contributors to total global mortality, such as cancer and cardiovascular diseases. We will put special attention on the latter.

Elevated plasma levels of cholesterol or hypercholesterolemia is a well-established risk factor for cardiovascular disease. Data on this are derived not only from studies performed in developed nations, but also from large studies involving low- and middle-income countries [2]. Accordingly, interventions to reduce blood lipid levels significantly reduce cardiovascular risk, and by far, the most efficient pharmacological approach to achieve cholesterol reduction has been the use of HMG-CoA reductase inhibitors, widely known as statins. For years, clinicians have relied on their efficacy and safety profile not only to reduce lipid levels, but also for primary and secondary cardiovascular prevention, which has been partly associated with their incompletely understood cholesterol-independent pleiotropic effects, mostly through reducing inflammation and improving vascular tone among others [3]. However, one of the main pitfalls of statin therapy corresponds to their well-documented variability in terms of LDL-C reduction, which is mainly seen in patients undergoing low-dose treatments. To date, besides several factors commonly known to affect response variability for example, age, race, gender, drug dose and genetic background among others, the molecular reasons underlying this outcome are still eluding the clear and solid evidence expected from the scientific community, which is the exact niche for a promising approach known as precision medicine, in other words, the right drug for the right person. The potential is vast - to individualize dosing and prevent adverse health outcomes, ultimately benefiting the user and decreasing public healthcare system expenditure by saving a significant amount of resources through the correction of previously failed therapies or the improvement of future treatments. 
In the field of statin therapy, huge efforts on a genome-wide scale have been performed to elucidate the genetic determinants of statin response [4-6]. Despite being necessary, the results have not been accompanied with the corresponding clinical success, confirming the role of a few previously associated genes and revealing an even smaller number of novel and potentially significant loci associated with statin interindividual or intraindividual differential response. Despite advancing the personalized medicine field, statin research is just not there yet, as the evidence from these large meta-analyses only contribute a residual overall explanation to statin response variability, generating at least two clear conclusions: the results from these studies still cannot be translated as they do not exceed a minimal threshold to be incorporated into the clinical decision-making process, which already is a matter of intense debate [7] and additional mechanisms may be involved. Nonetheless, obstacles found and foreseen in the translation of pharmacogenomic knowledge to bedside led to the creation of the Clinical Pharmacogenetics Implementation Consortium (CPIC) [8], a nonprofit international organization aimed at developing standardized guidelines on the use of genomic data, pushing forward the incipient arrival of precision medicine to clinical care.

Remarkably, the pioneer investigations performed by the Nobel Laureates in Physiology or Medicine, Michael S Brown and Joseph L Goldstein, disclosing the LDLR pathway of cholesterol metabolism [9] are still tremendously valid, even though they were reported more than three decades ago. With solid precision, the advance of science boundaries makes it possible to fuse the new lessons obtained from miRNA research with the old lessons taught from the LDLR pathway in an effort to offer another explanation to fill the current gap regarding response variability of statin treatment. Why? As widespread as they can be, miRNAs not only play a role, but are essential for the regulation of crucial genes implicated in the molecular pathway formerly described by Brown and Goldstein, which has been clearly demonstrated using both in vitro and in vivo models [10,11]. But do statins take part in the interplay between miRNAs and their target genes? We and others have reported positive findings on this issue [12,13], suggesting that, for instance, miRNAs can be modulated in subjects with hypercholesterolemia, and that the modulation is dependent on the type of statin employed [14], meaning that miRNAs are likely to be implicated in the differential response observed with different statin treatments.

At this point in time, the emerging field of miRNAs and statin research is experiencing a strong interest with exceptional results. Mutations of a previously unknown protein termed PCSK9 causing autosomal dominant hypercholesterolemia [15] revealed a new main character to be added to the well described LDLR pathway in cholesterol regulation. Moreover, subsequent studies on this seminal breakthrough revealed PCSK9 gain-of-function and loss-of-function variations associated with autosomal dominant hypercholesterolemia and low cholesterol levels, respectively. Thus, the protein function - to cleave LDLR - makes PCSK9 a possible target to be therapeutically manipulated. In fact, it has been recently demonstrated that a number of miRNAs control PCSK9 [16], but a significant amount of accumulated knowledge makes the plot thicker. As above mentioned, statins are the preferred lipid-lowering drug to decrease LDL-C, but one of the reasons that statins may not be fully capable to reduce cholesterol levels with a superior efficiency is that, besides increasing LDLR to remove LDL-C from circulation, statins also increase PCSK9-related lysosomal LDLR degradation, which could be especially important when using medium to high intensity statin doses [17]. Yet, a 2015 report concluded that both LDLR and PCSK9 pathways could be therapeutically manipulated by inhibiting miRNA-27a, an miRNA that normally decreases LDLR while increasing both mRNA and secreted PCSK9 [18]. Hence, various studies support the feasibility of considering miRNAs as therapeutic targets for $\mathrm{FH}$, a scenario that has already reached clinical impact [19]. Consequently, the use of miRNAs as PCSK9 inhibitors is considered nowadays a practical therapeutic approach [20], having in mind the recent US FDA approval of Evolocumab and Alirocumab, two novel medications for LDL-C reduction based on PCSK9 inhibition.

Finally, is the massive task of making precision medicine a reality completely within our reach? To know what we do not know, a large road remains ahead, as a growing number of continuous breakthroughs demonstrate us that accumulated knowledge so far can only expand, especially in the postgenomic era. However, and without a doubt, the most enlightening path is to continue research, accelerating its translation into clinical practice.

Financial \& competing interests disclosure

The authors have no relevant affiliations or financial involvement with any organization or entity with a financial interest in or financial conflict with the subject matter or materials discussed in the manuscript. This includes employment, consultancies, honoraria, stock ownership or options, expert testimony, grants or patents received or pending, or royalties.

No writing assistance was utilized in the production of this manuscript. 


\section{References}

1 Lee RC, Feinbaum RL, Ambros V. The C. elegans heterochronic gene lin-4 encodes small RNAs with antisense complementarity to lin-14. Cell 75(5), 843-854 (1993).

2 Yusuf S, Hawken S, Ounpuu S et al. Effect of potentially modifiable risk factors associated with myocardial infarction in 52 countries (the INTERHEART study): case-control study. Lancet 364(9438), 937-952 (2004).

3 Oesterle A, Laufs U, Liao JK. Pleiotropic effects of statins on the cardiovascular system. Circ. Res. 120(1), 229-243 (2017).

4 Barber MJ, Mangravite LM, Hyde CL et al. Genome-wide association of lipid-lowering response to statins in combined study populations. PLoS ONE 5(3), e9763 (2010).

5 Postmus I, Trompet S, Deshmukh HA et al. Pharmacogenetic meta-analysis of genome-wide association studies of LDL cholesterol response to statins. Nat. Commun. 5, 5068 (2014).

6 Postmus I, Warren HR, Trompet S et al. Meta-analysis of genome-wide association studies of HDL cholesterol response to statins. J. Med. Genet. 53(12), 835-845 (2016).

7 Grosse SD, Khoury MJ. What is the clinical utility of genetic testing? Genet. Med. 8(7), 448-450 (2006).

8 Relling MV, Klein TE. CPIC: Clinical Pharmacogenetics Implementation Consortium of the Pharmacogenomics Research Network. Clin. Pharmacol. Ther. 89(3), 464-467 (2011).

9 Brown MS, Goldstein JL. A receptor-mediated pathway for cholesterol homeostasis. Science 232(4746), 34-47 (1986).

10 Najafi-Shoushtari SH, Kristo F, Li Y et al. microRNA-33 and the SREBP host genes cooperate to control cholesterol homeostasis. Science 328(5985), 1566-1569 (2010).

11 Goedeke L, Wagschal A, Fernandez-Hernando C, Naar AM. miRNA regulation of LDL-cholesterol metabolism. Biochim. Biophys. Acta 1861(12 Pt B), 2047-2052 (2016).

12 Niesor EJ, Schwartz GG, Perez A et al. Statin-induced decrease in ATP-binding cassette transporter A1 expression via microRNA33 induction may counteract cholesterol efflux to high-density lipoprotein. Cardiovasc. Drugs Ther. 29(1), 7-14 (2015).

13 Zambrano T, Hirata RDC, Hirata MH, Cerda A, Salazar LA. Altered microRNome profiling in statin-induced HepG2 cells: a pilot study identifying potential new biomarkers involved in lipid-lowering treatment. Cardiovasc. Drugs Ther. 29(6), 509-518 (2015).

14 Zambrano T, Hirata RDC, Hirata MH, Cerda A, Salazar LA. Statins differentially modulate microRNAs expression in peripheral cells of hyperlipidemic subjects: a pilot study. Eur. J. Pharm. Sci. 117, 55-61 (2018).

15 Abifadel M, Varret M, Rabes JP et al. Mutations in PCSK9 cause autosomal dominant hypercholesterolemia. Nat. Genet. 34(2), $154-156$ (2003).

16 Naeli P, Mirzadeh Azad F, Malakootian M, Seidah NG, Mowla SJ. Post-transcriptional regulation of PCSK9 by miR-191, miR-222, and miR-224. Front. Genet. 8, 189 (2017).

17 Welder G, Zineh I, Pacanowski MA, Troutt JS, Cao G, Konrad RJ. High-dose atorvastatin causes a rapid sustained increase in human serum PCSK9 and disrupts its correlation with LDL cholesterol. J. Lipid Res. 51(9), 2714-2721 (2010).

18 Alvarez ML, Khosroheidari M, Eddy E, Done SC. microRNA-27a decreases the level and efficiency of the LDL receptor and contributes to the dysregulation of cholesterol homeostasis. Atherosclerosis 242(2), 595-604 (2015).

19 Bouchie A. First microRNA mimic enters clinic. Nat. Biotechnol. 31(7), 577 (2013).

20 Momtazi AA, Banach M, Pirro M, Stein EA, Sahebkar A. microRNAs: new therapeutic targets for familial hypercholesterolemia? Clin. Rev. Allergy Immunol. 54(2), 224-233 (2018). 
